\title{
Central Projections of the Lateral Line Nerves in the Shovelnose Sturgeon
}

\author{
JOHN G. NEW AND R. GLENN NORTHCUTT \\ Division of Biological Sciences, University of Michigan, Ann Arbor, Michigan 48109
}

\begin{abstract}
Primary projections of the anterior (ALLN) and posterior (PLLN) lateral line nerves were traced in the shovelnose sturgeon by means of horseradish peroxidase (HRP) histochemistry and silver degeneration. The trunk of the ALLN divides into dorsal and ventral roots as it enters the medulla. Fibers of the dorsal root form ascending and descending branches that terminate within the ipsilateral dorsal octavolateralis nucleus and the dorsal granular component of the lateral eminentia granularis. Fibers of the ventral root of the ALLN, as well as fibers of the PLLN, enter the medulla ventral to the dorsal root of the ALLN where some of the fibers terminate among the dendrites of the magnocellular octaval nucleus. The bulk of the fibers form ascending and descending branches that terminate within the ipsilateral medial octavolateralis nucleus. A portion of the ascending fibers continue more rostrally and terminate in the ipsilateral eminentia granularis and bilaterally in the cerebellar corpus. Some fibers of the descending rami of both the ALLN and PLLN extend beyond the caudal limit of the medial octavolateralis nucleus to terminate in the caudal octavolateralis nucleus. The HRP cases also revealed retrogradely filled large neurons whose axons course peripherally in the lateral line nerve and are likely efferent to the lateral line organs.
\end{abstract}

Key words: HRP histochemistry, lateral line system, sturgeons, electroreception, brainstem

Lateral line receptors in chondrostean bony fishes (sturgeons and paddlefish), like those in chondrichthian fishes, comprise electroreceptive ampullae that are restricted to the head and mechanoreceptive neuromasts distributed over the head and the rest of the body (Jørgensen et al., '72; Weizel, '78; Jørgensen, '80; Teeter et al., '80). In both chondrichthian and chondrostean fishes, the electroreceptors and mechanoreceptors of the head are innervated by anterior lateral line nerves, and the mechanoreceptors of the trunk and tail are innervated by posterior lateral line nerves (Murray, '60; Marinelli and Strenger, '73).

Recent anatomical and physiological studies of chondrichthian fishes demonstrate segregation of lateral line nerve fibers carrying electroreceptive and mechanoreceptive information (Boord and Campbell, '77; Koester and Boord, '78; Bodznick and Northcutt, '80; Koester, '83). In these fishes, the anterior lateral line nerve is divided into dorsal and ventral roots as it enters the medulla. The dorsal root is composed of electroreceptive fibers that terminate in the dorsal octavolateralis nucleus of the medulla, whereas the ventral root of the anterior lateral line nerve and the pos- terior lateral line nerve consist of mechanoreceptive fibers that terminate in the medial octavolateralis nucleus.

Sturgeons and paddlefishes also possess an anterior lateral line nerve divided into dorsal and ventral roots, as well as a lateralis area of the medulla divided into dorsal and medial octavolateralis nuclei (Johnston, '01; Hocke Hoogenboom, '29; Pearson, '36; Larsell, '67; McCormick, ' 82 ). The similarities in electroreceptor distribution and the peripheral organization of the anterior lateral line nerve, and the lateral line area of the medulla in chondrichthian and chondrostean fishes strongly support the contention that electroreceptive components of the lateral line nerves are organized in a similar manner and were probably inherited from the common ancestor of chondrichthian and bony fishes (McCormick, ' 82 ). In order to test this hypothesis, the primary projections of the lateral line nerves in the shovel-

Accepted November 30, 1983.

Dr. John G. New's present address is Department of Biology, Wesleyan University, Middletown, CT 06457. 
nose sturgeon were determined by horseradish peroxidase histochemistry and axon degeneration techniques.

\section{MATERIALS AND METHODS}

Specimens of both sexes of shovelnose sturgeon, Scaphirhynchus platorynchus, were collected from the Mississippi River (E. Saeugling, Guttenberg, Iowa) and housed in freshwater aquaria at $14^{\circ} \mathrm{C}$ for approximately 1 week prior to surgery.

Two experimental neuroanatomical procedures were used to elucidate the primary projections of the lateral line nerves: horseradish peroxidase histochemistry and axonal degeneration. Fish were anesthetized by immersion in a $0.025 \%$ solution of tricaine methanesulfonate (MS222) and kept moist throughout the course of the operation. It was unnecessary to perfuse the gills with aerated water during surgery, which required less than an hour, and the fish recovered quickly when returned to the home aquaria.

For the HRP cases, an anterior (six cases) or posterior (nine cases) lateral line nerve was exposed close to its entry into the medulla and transected. The entire nerve was transected in the anterior lateral line nerve cases as the dorsal and ventral roots of this nerve form only immediately prior to entering the medulla. A pledget of gelfoam saturated with $40 \%$ horseradish peroxidase (Sigma VI) was placed on the proximal stump of the transected nerve; the wound was packed with gelfoam and sealed with dental acrylic. After survival times of 6-16 days, the animals were reanesthetized and perfused transcardially with cold $0.1 \mathrm{M}$ phosphate buffer ( $\mathrm{pH} 7.4$ ), followed by $2 \%$ glutaraldehyde in $0.1 \mathrm{M}$ phosphate buffer. The brains were then removed from the neurocranium and fixed for an additional 2-4 hours and washed in $0.1 \mathrm{M}$ phosphate buffer with $10 \%$ sucrose. The meninges were removed, and the brains were embedded in gelatin blocks and fixed for an additional 4 hours in $2 \%$ glutaraldehyde in sucrose buffer. Thirty- to 35 micron transverse sections were cut on a sled microtome with a freezing attachment, collected in phosphate buffer, and stored at $7^{\circ} \mathrm{C}$. The sections were reacted with either $3,3^{\prime}, 5,5^{\prime}$ tetramethylbenzidine, following the method of $\mathrm{Me}$ sulam ('78), or o-dianisidine dihydrochloride, following the method of Coleman et al. (76). After processing, the sections were mounted on chrome-alum slides and lightly counterstained with neutral red.

For the axonal degeneration cases, an anterior (two cases) or posterior (two cases) lateral line nerve was transected proximal to the ganglion. The wound was then packed with gelfoam and sealed with dental acrylic. After a survival time of 14 days, the animals were reanesthetized and perfused transcardially with $0.1 \mathrm{M}$ phosphate buffer, followed by $10 \%$ formalin. The brains were embedded in gelatin, sectioned at $30-33 \mu \mathrm{m}$ on a freezing microtome, and stained by the Wiitanen ('69) modification for revealing degenerating axons and terminals.

Tracings of the sections were made with the aid of a Bausch and Lomb microprojector and a Leitz Wetzlar Ortholux II microscope with camera lucida attachment. The location and charting of the specific nuclei in the brainstem were accomplished with the aid of transverse sections, serially mounted and stained by the Bodian method.

\section{RESULTS \\ Organization of the octavolateralis nuclei}

The octavolateralis area in Scaphirhynchus (Fig. 1) consists of a number of medullary nuclei, extending caudally from approximately the level of entrance of the trigeminal nerve to the level of the obex. The nuclei of the octavolateralis area are arranged in three longitudinal columns (Fig. 1). The dorsal octavolateralis nucleus is situated dorsal to the medial octavolateralis nucleus and the caudal octavolateralis nucleus, both of which occupy a position dorsal to the octaval nuclei.

The dorsal octavolateralis nucleus appears as an elongated ridge directly overlying the dorsal surface of the cerebellar crest. Its rostral terminus appears as an abrupt ridge at the notch of the lateral lobule of the cerebellum, and it extends caudally to the level of entry of the PLLN (Figs. 1, 2D, 3A,B). The dorsal nucleus consists of polygonal, bipolar, and granular cells, as well as a population of Purkinje-like cells located along the ventral border of the nucleus. The dendrites of the Purkinje-like cells extend ventrally into the neuropil of the cerebellar crest and dorsally into the dorsal nucleus itself where they synapse among incoming fibers. Distinct bundles of efferent fibers (unpublished observations) pass from the ventromedial border of the dorsal nucleus, around the medial surface of the cerebellar crest, and past the medial border of the medial octavolateralis nucleus. The efferents of the octavolateralis nucleus (arcuate fibers) are also very distinct, extending from the region around the medial nucleus to the midline and decussating to the contralateral basal region (Fig. 3AC).

The medial octavolateralis nucleus extends rostrocaudally from approximately the level of entrance of the trigeminal nerve to the level of entrance of the vagus nerve (Figs. 1, 2D, 3A-C). The medial nucleus consists of polygonal, bipolar, and granular cells, as well as a dorsal layer of Purkinje-like cells similar to those in the dorsal octavolateralis nucleus. These cells are situated along the dorsal border of the medial nucleus and, as in the dorsal nucleus, maintain a primarily vertical orientation, extending dendrites dorsally into the cerebellar crest and ventrally into the body of the medial nucleus. The cerebellar crest is a dense neuropil, continuous with the molecular layer of the cerebellum. Unlike the cerebellar crest of elasmobranchs, it is a single fused mass overlying the dorsal surface of the medial nucleus and extending caudally to a point slightly rostral to the obex.

The cells of the medial octavolateralis nucleus are replaced caudally by the smaller, spindle-shaped cells of the caudal octavolateralis nucleus at a point rostral to the anterior rootlets of the vagus nerve (Figs. 1, 3D). The cells of the caudal nucleus extend caudally to a point rostral to the level of the obex where they are replaced by the dense fibers of the funicular nucleus.

Scaphirhynchus possesses four medullary octaval nuclei. The anterior octaval nucleus consists of granular, multipolar, and fusiform cells and extends from a rostral terminus at the level of entrance of the trigeminal nerve caudally to a level slightly rostral to the level of entrance of the anterior lateral line nerves (ALLN; Figs. 1, 2D). The magnocellular octaval nucleus extends from a point at the caudal level of entrance of the ALLN to terminate just caudal to the entrance of the octaval nerve. The magnocellular nucleus consists primarily of large and very distinct multipolar cells situated at the ventromedial border of the medial octavolateralis nucleus and the ventrolateral border of the vagal lobe (Figs. 1, 3A,B). A pair of very large Mauthner cells appears bilaterally at a point slightly caudal to the magnocellular nucleus. These very distinct multipolar cells 


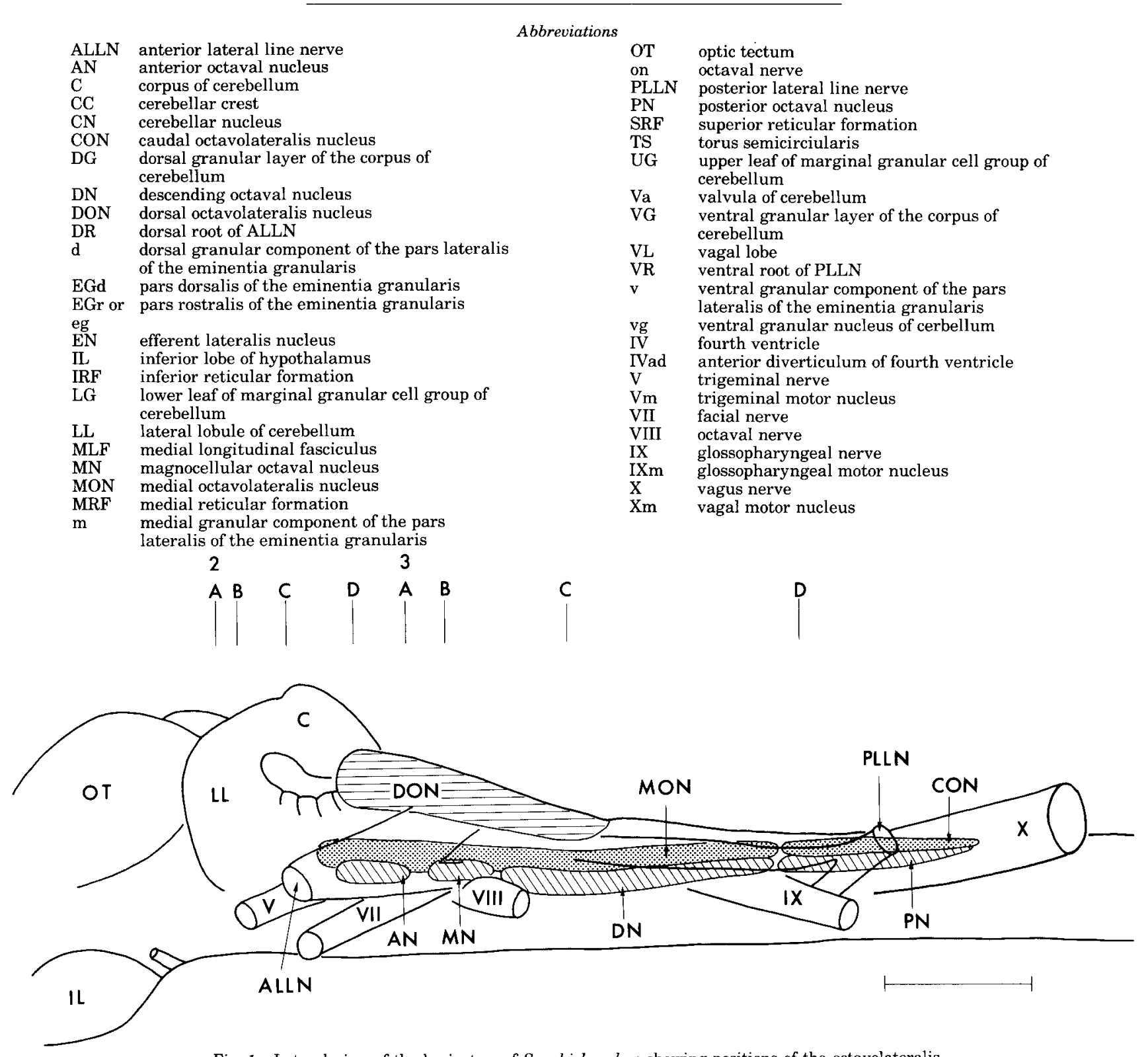

Fig. 1. Lateral view of the brainstem of Scaphirhynchus showing positions of the octavolateralis nuclei as they would appear projected onto the lateral surface of the brain.

lie immediately under the dorsal surface of the basal plate of the medulla, close to the midline, and slightly lateral to the medial longitudinal fasciculi.

The descending octaval nucleus extends caudally from a point slightly caudal to the magnocellular nucleus to the level of entrance of the glossopharyngeal nerve. It consists of large fusiform and granular cells and is positioned directly ventrolateral to the medial octavolateralis nucleus (Figs. 1, 3C).

The posterior octaval nucleus extends caudally from the posterior terminus of the descending octaval nucleus to a level slightly rostral to the obex. The cells of the posterior nucleus are not readily discernible from those of the caudal octavolateralis nucleus dorsomedially adjacent (Figs. 1, 3D)

All of the octaval nuclei are located ventral to the medial and caudal octavolateralis nuclei (Fig. 1). The anterior oc- taval nucleus is directly ventral to the medial octavolateralis nucleus (Fig. 2D); the magnocellular nucleus is more ventromedial to the medial octavolateralis nucleus (Fig. 3 $\mathrm{A}, \mathrm{B})$, and the descending and posterior octaval nuclei are progressively more ventrolateral (Fig. 3C,D).

\section{Organization of the cerebellum}

The cerebellum of Scaphirhynchus consists of an unpaired medial body extending ventrally and rostrally into the fourth ventricle, and the associated lateral lobules which extend laterally and dorsally. The dense, fibrous medial layer of the corpus of the cerebellum is continuous with that of the valvula, rostrally, and with the lateral lobules.

Within the dorsolateral portion of the cerebellum there are two distinct granular cell masses. The dorsolateral cell group, less densely packed than the ventromedial group, is 

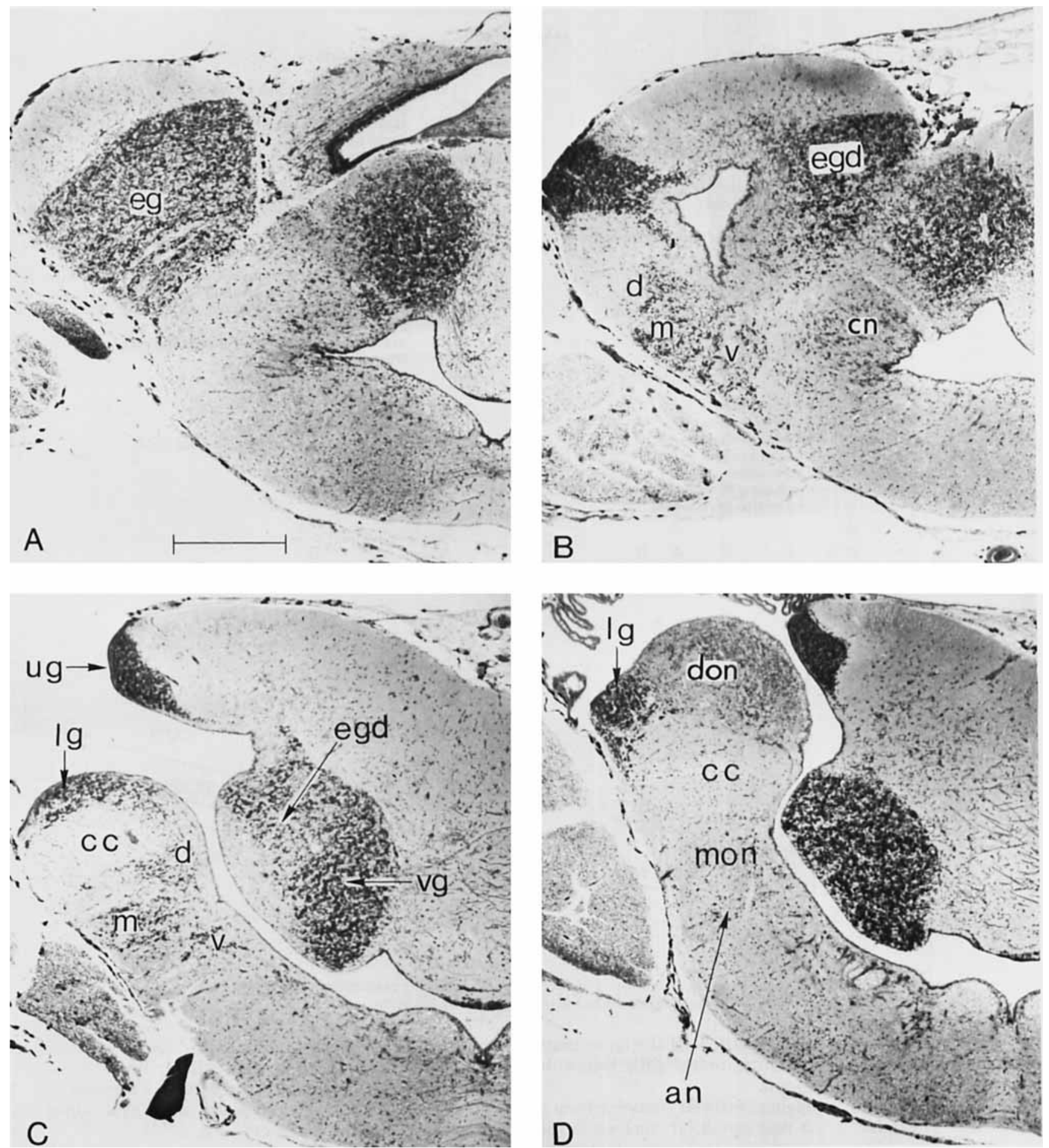

Fig. 2. Photomicrographs of transverse Bodian-stained sections of the cerebellum and octavolateralis nuclei in Scaphirhynchus at the level indicated in Figure 1. Bar scale equals $1 \mathrm{~mm}$

continuous with the eminentia granularis pars dorsalis. The granular cells of the ventromedial group are termed the ventral granular layer of the cerebellar corpus and extend rostrally into the valvula (Figs. 4A-D, 5A,B). Another mass of densely packed granular cells appears bilaterally, dorsal to the valvular fissure; this dorsal granular layer also extends rostrally into the valvula (Fig. 4A), directly dorsal to the ventral granular layer.

\section{Organization of the eminentia granularis}

The eminentia granularis in Scaphirhynchus is well developed and occupies a large portion of the lateral lobules 

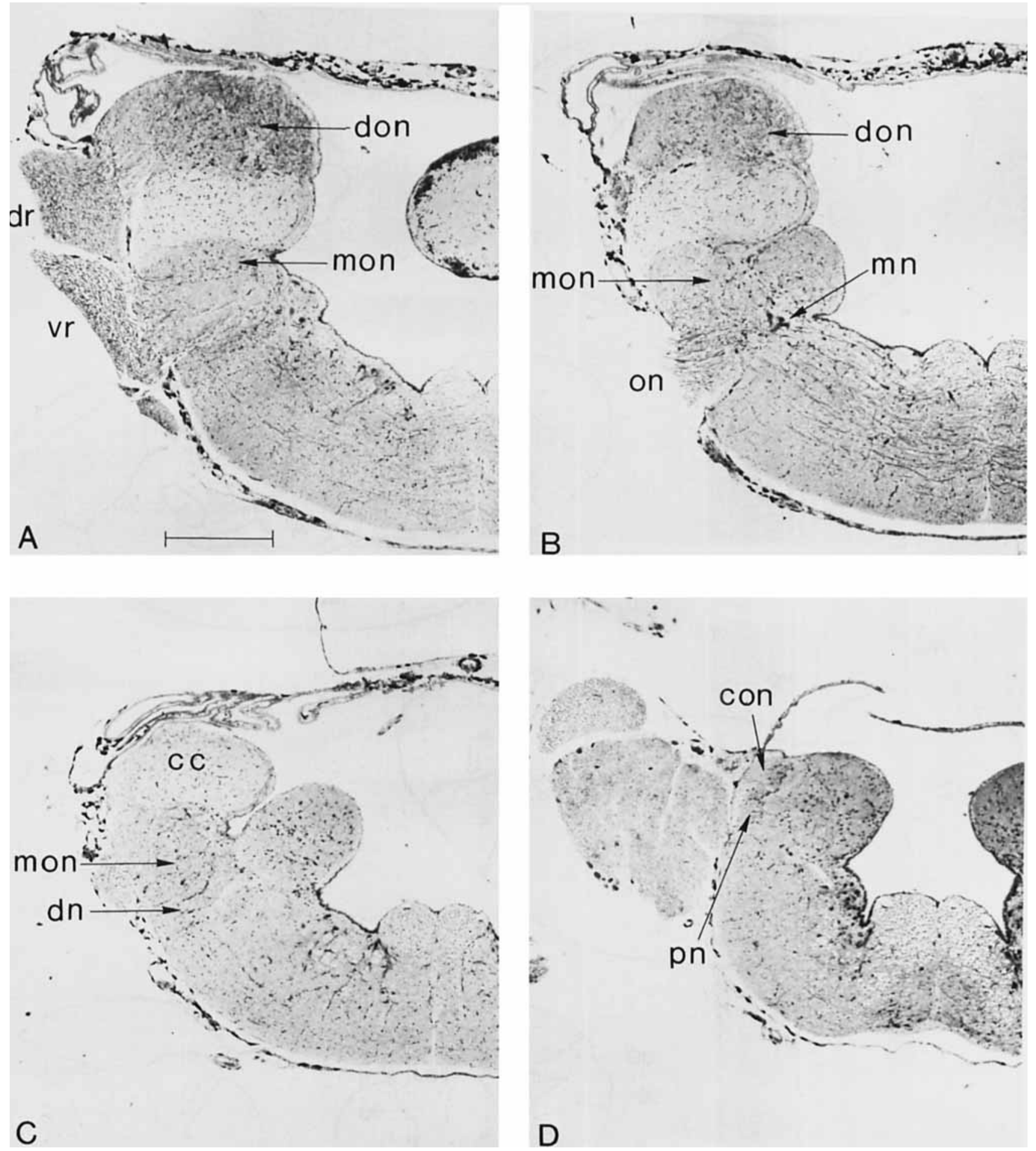

Fig. 3. Photomicrographs of transverse Bodian-stained sections through the octavolateralis region in Scaphirhynchus at the levels indicated in Figure 1. Bar scale equals $1 \mathrm{~mm}$.

of the cerebellum (Fig. 1). It extends from an area quite caudal in the corpus of the cerebellum, rostrally and laterally through the lateral lobules, then ventrally and caudally to the anterior borders of the octavolateralis and octaval nuclei. We divide the eminential granularis into three major subdivisions: eminentia granularis pars dor- salis, eminentia granularis pars rostralis, and eminentia granularis pars lateralis, with dorsal, medial, and ventral components.

The eminentia granularis pars dorsalis becomes visible in the caudal portion of the corpus of the cerebellum, appearing directly dorsolateral to the ventral granular layer 

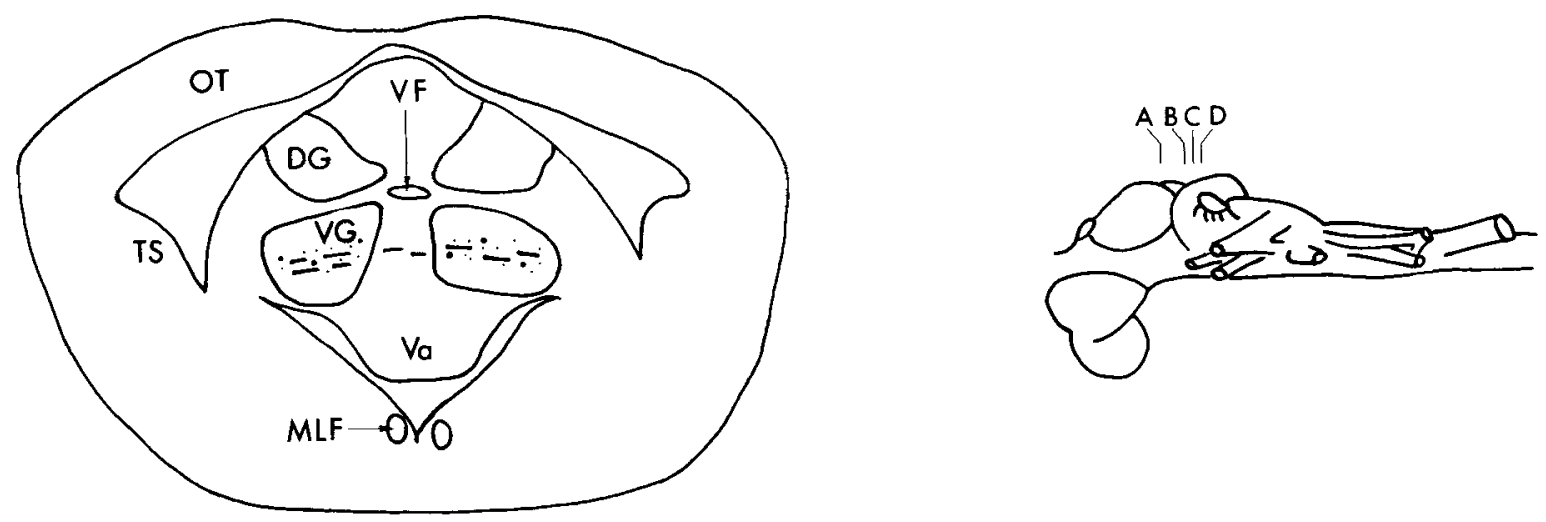

A

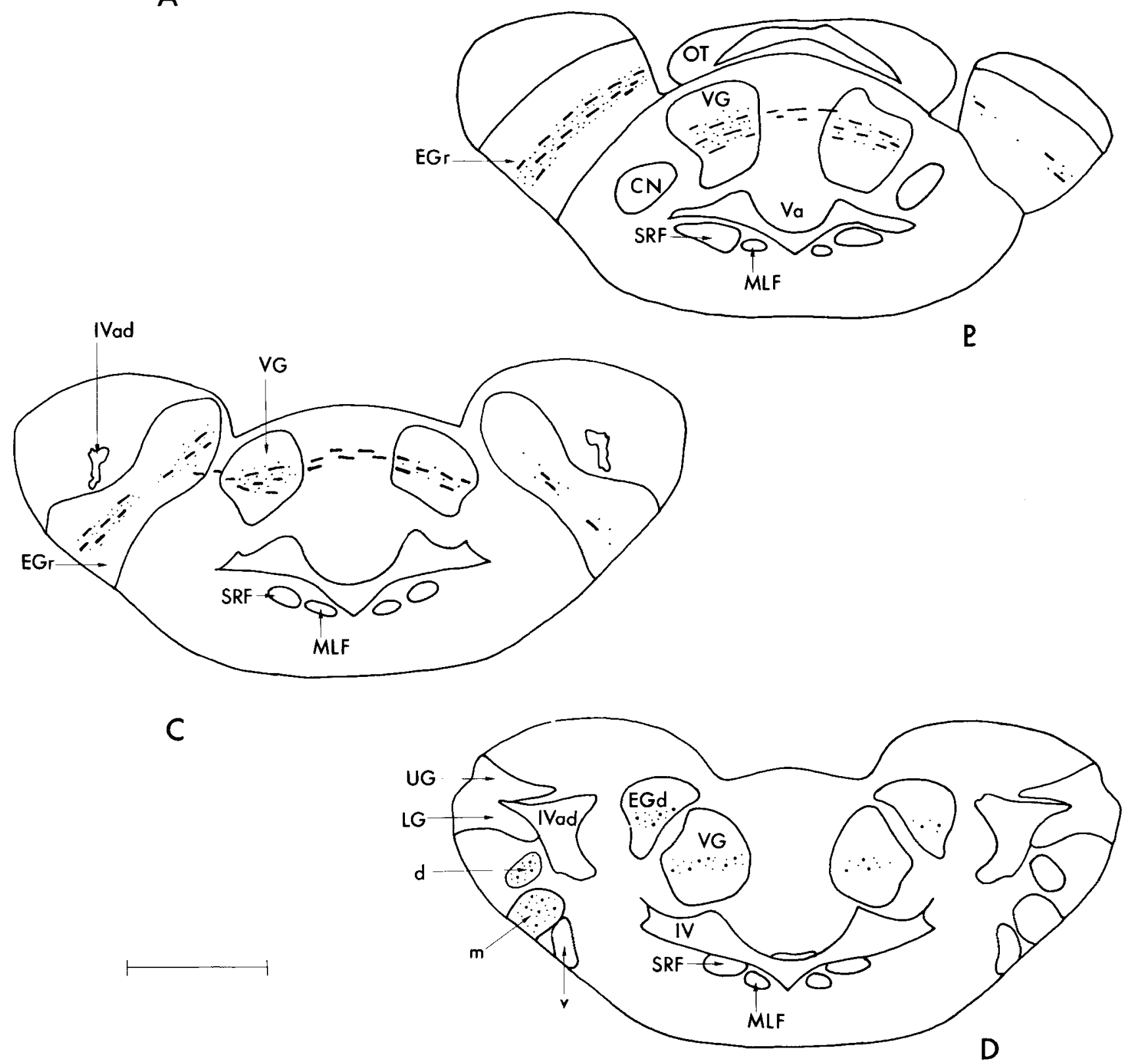

Fig. 4. Line drawings of transverse sections of the octavolateralis region showing the central projections of the anterior lateral line nerve. Dashed lines and heavy dots indicate HRP-labelled axons. Light stippling indicates terminals. Bar scale equals $1 \mathrm{~mm}$. 

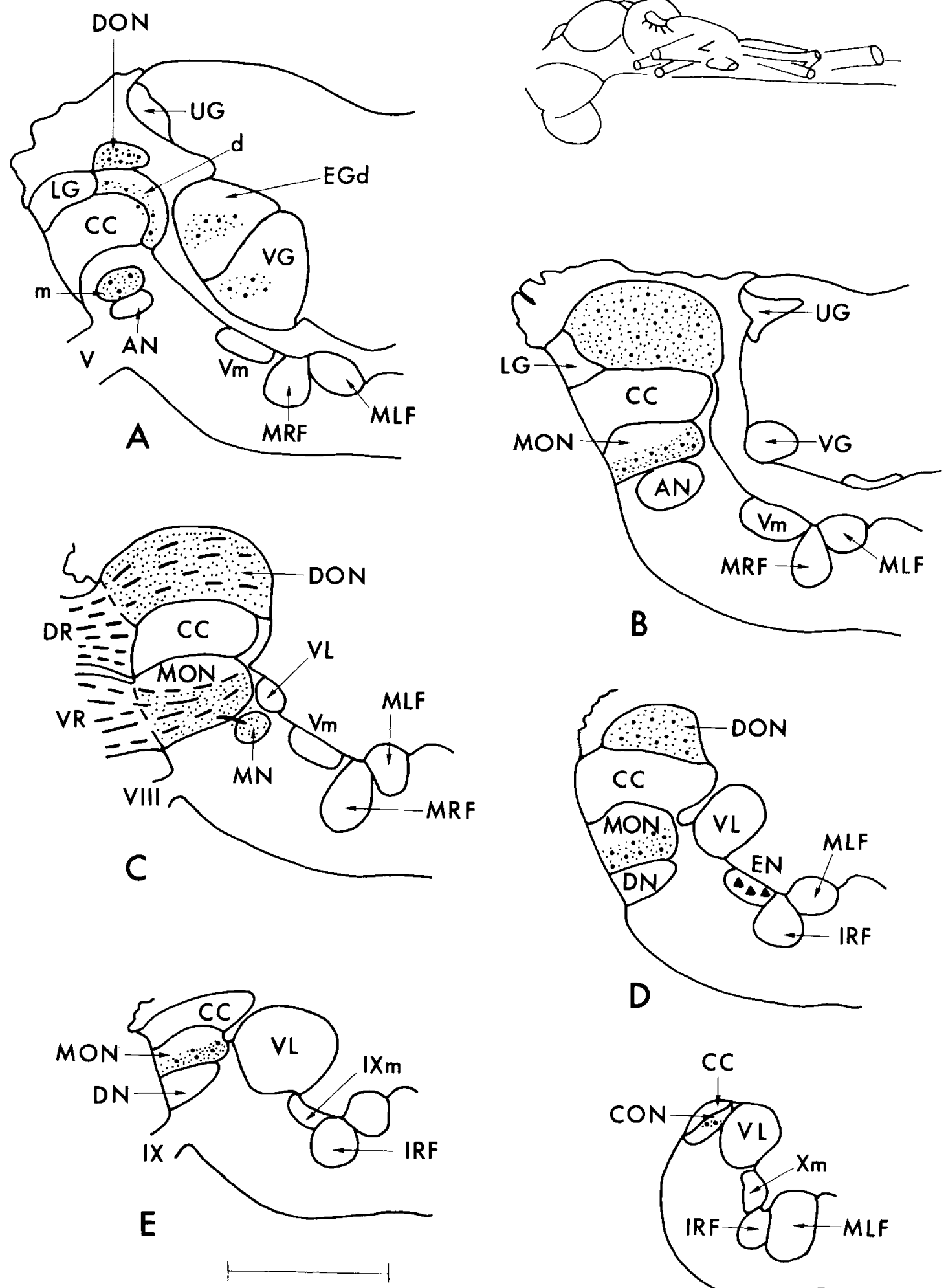

Fig. 5. Line drawings of transverse sections of octavolateralis region, showing the projections of the anterior lateral line nerve. Dashed lines and heavy dots indicate HRP-labelled axons, light stippling indicates terminals. Filled triangles indicate retrogradely filled cell bodies. Bar scale equals $1 \mathrm{~mm}$. 

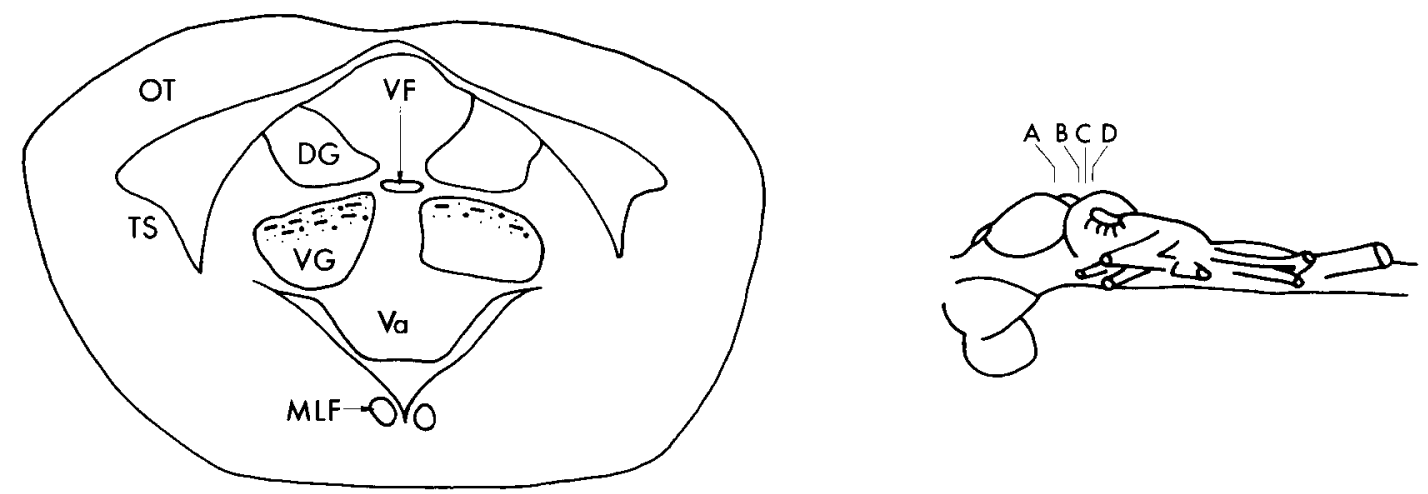

A
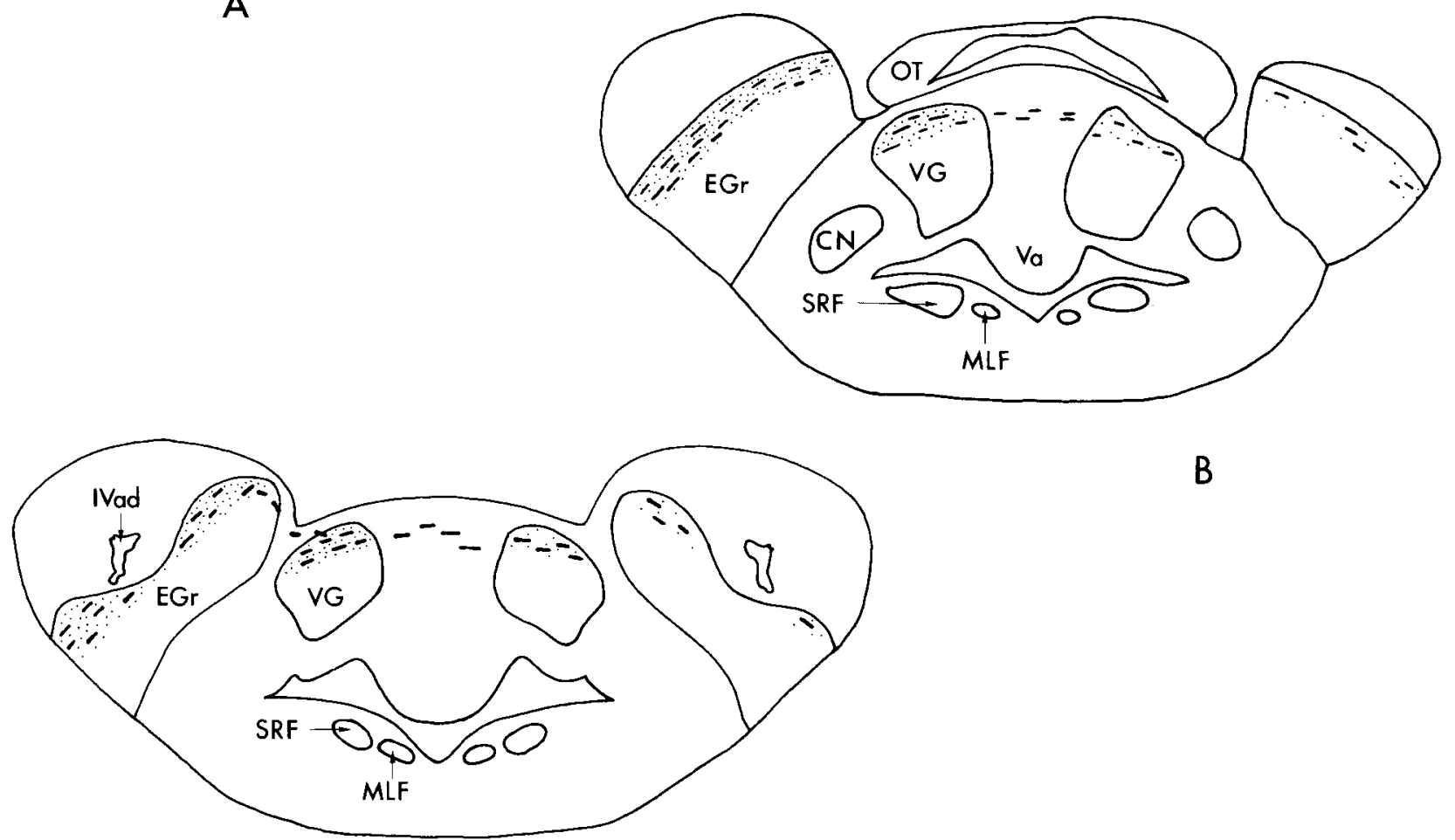

B

C

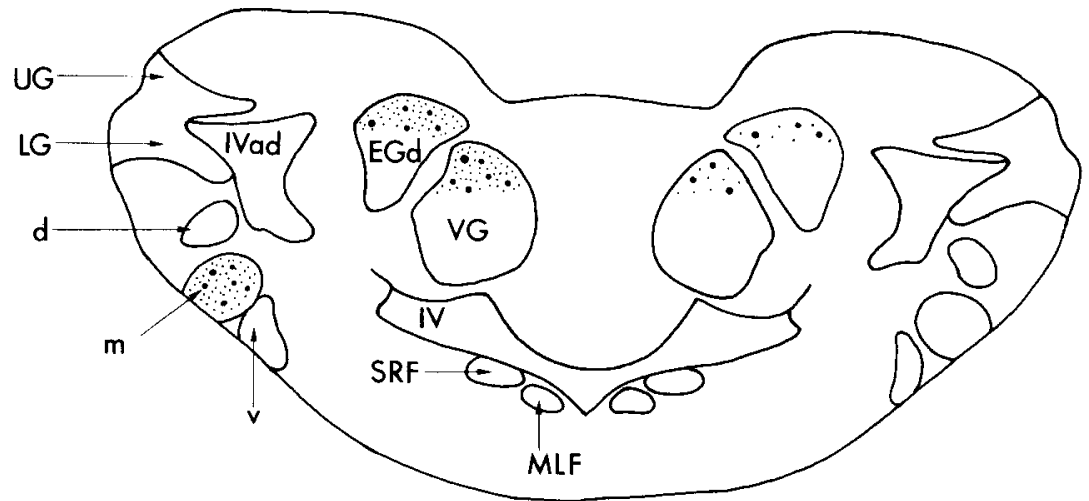

$\longmapsto-1$

D

Fig. 6. Line drawings of transverse sections of the octavolateralis area showing the projections of the posterior lateral line nerve. Dashed lines and heavy dots indicate HRP-labelled axons, light stippling indicates terminals. Bar scale equals $1 \mathrm{~mm}$. 

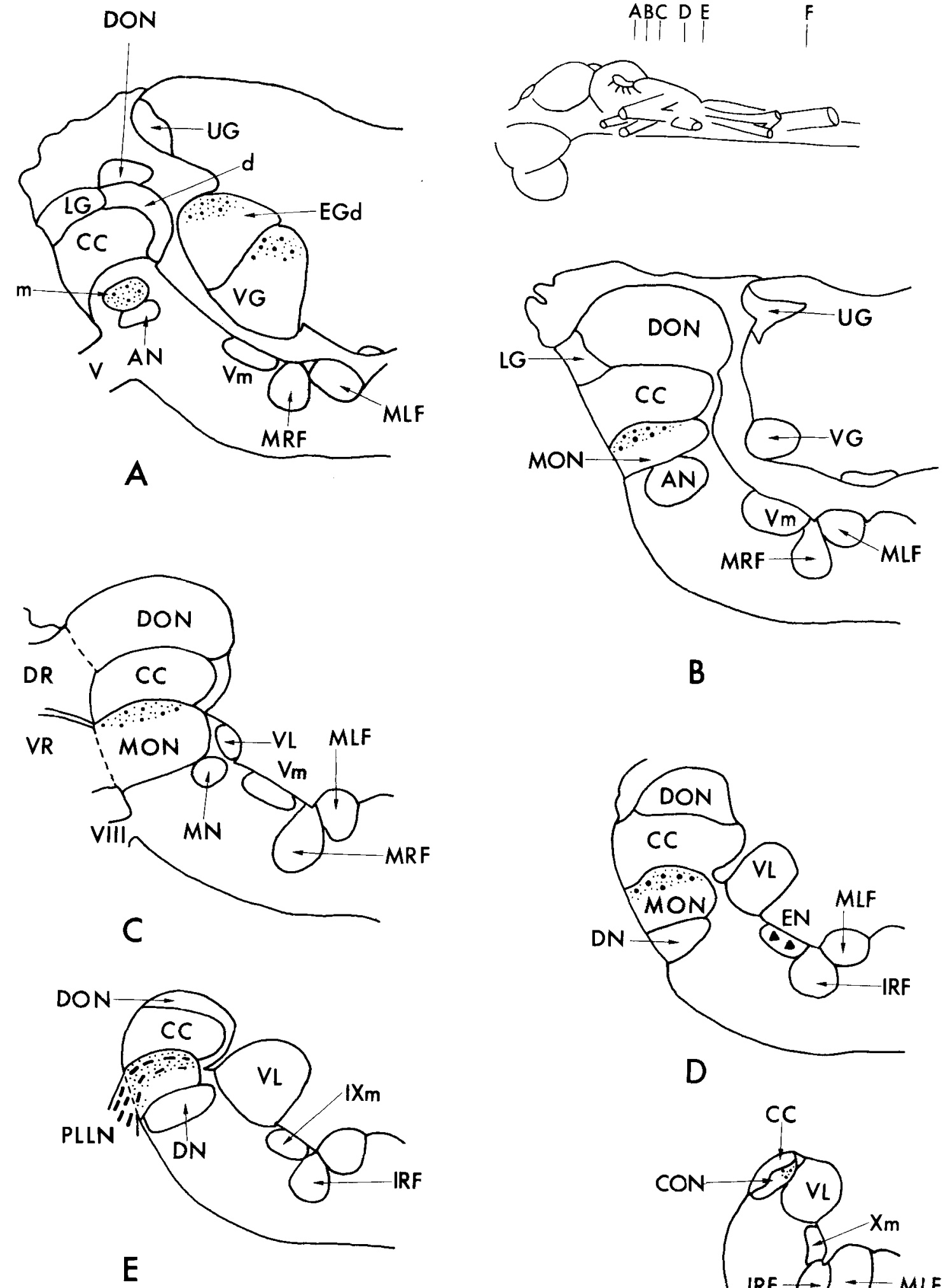

B
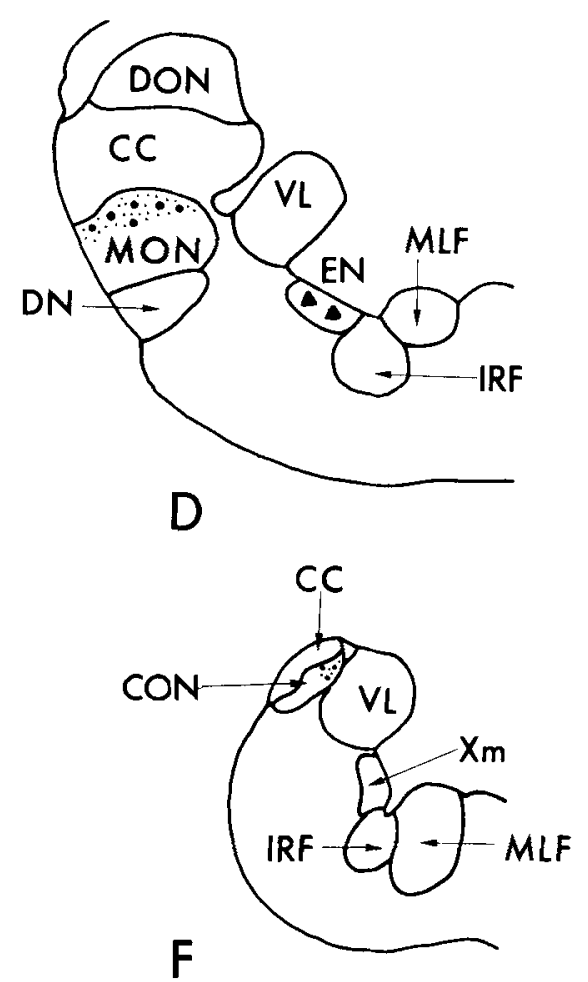

Fig. 7 Line drawings of transverse sections through the octavolateralis area showing the projections of the posterior lateral line nerve. Dashed lines and heavy dots indicate HRP-labelled axons, light stippling indicates terminals. Filled triangles indicate retrogradely filled cell bodies. (See Fig. 5.) Bar scale equals $1 \mathrm{~mm}$. 
of the cerebellar corpus (Fig. 5A). It is distinguishable from the cells of the latter, which are larger and more densely packed. The eminentia granularis pars dorsalis extends rostrally and somewhat dorsolaterally (Fig. 4D), to be replaced by the pars rostralis which begins at that point where the cells of the eminentia spread laterally to form a wide band across the lateral lobule, anterior to the rostral terminus of the anterior diverticulum of the fourth ventricle. The pars rostralis extends rostrally to the anterior terminus of the lateral lobule (Fig. 4B,C). The eminentia granularis pars lateralis extends caudally from the ventrolateral portion of the pars rostralis and forms three components: dorsal, medial, and ventral (Figs. 4D, 5A). The ventral component of the pars lateralis extends caudally to end among the cells at the rostral border of the anterior octaval nucleus; the medial component of the pars lateralis ends in a similar manner among the anterior cells of the medial octavolateralis nucleus. The dorsal component of the pars lateralis extends caudally, slightly dorsomedial to the medial and ventral components (Fig. 4D), and then can be traced dorsomedially around the medial surface of the cerebellar crest, ending on its dorsomedial surface (Fig. 5A). The caudal level at which the dorsal component ends is directly medial to the lower leaf of the marginal granular layer of the lateral lobule, and it is distinguishable from the latter by its slightly smaller, less densely packed cells (Figs. 2C, 5A). The cells of the dorsal component of the pars lateralis are replaced caudally by the rostral cells of the dorsal octavolateralis nucleus.

\section{Central projections of the lateralis nerves}

The central projections of the anterior and posterior lateral line nerves are predominantly ipsilateral and form ascending and descending components within the brainstem. The course and termination of these nerves are charted in Figures 4 through 7, based on two HRP cases that are representative of other HRP preparations and degeneration cases.

Fibers of the dorsal root of the ALLN enter the dorsal octavolateralis nucleus (Fig. 5C) and bifurcate into ascending and descending branches that extend throughout the dorsal nucleus, terminating among the cells of that nucleus (Figs. 4, 5, 8A). A small group of fibers extends rostrally beyond the anterior border of the dorsal nucleus and terminates in the dorsal granular component of the eminentia granularis pars lateralis (Fig. 5A). Fibers of the dorsal root of the ALLN do not appear to reach the ventral granular layer of the cerebellar corpus or decussate to the contralateral layer, as do the fibers of the ventral root.

Fibers of the ventral root of the ALLN enter the medial nucleus where they also form ascending and descending tracts and their terminals are restricted to the ventral portion of the medial octavolateralis nucleus. A small group of fibers also terminate within the magnocellular octaval nucleus (Fig. 5C). Other fibers of the descending tract continue caudally into the caudal octavolateralis nucleus and terminate within the ventral portion of this nucleus (Fig. 5F). Fibers of the ascending tract continue rostrally beyond the medial octavolateralis nucleus into a medial component of the eminentia granularis pars lateralis where some of these fibers appear to terminate (Fig. 5A). Other fibers continue more rostrally in the pars rostralis, then caudally and dorsally through the pars dorsalis, terminating throughout the entire eminentia granularis (Figs. 4B-D, 5A). The experimental cases also revealed fibers extending medially to the ventral granular layer of the cerebellar corpus (Fig. 4C), bifurcating into ascending and descending branches that extend throughout the ventral granular layer of the corpus caudally and the valvula rostrally. A number of fibers decussate across the dense molecular layer of the corpus of the cerebellum to the contralateral ventral granular layer and the contralateral eminentia granularis pars dorsalis and rostralis, terminating within these cell groups (Figs. $5 \mathrm{~A}, 4)$.

Within the ipsilateral and contralateral eminentiae granularis and ventral granular layer of the cerebellar corpus, the fibers of the ALLN remain strictly confined to the medial portions of those cell groups, reflecting a definite topographic organization of anterior lateralis afferents within the cerebellum as well as the brain stem.

The posterior lateral line nerve (PLLN) enters the lateral wall of the medulla at approximately the level of the caudal border of the dorsal octavolateralis nucleus (Fig. 7E). Fibers of the PLLN directly enter the dorsal portion of the medial octavolateralis nucleus and form ascending and descending branches. Descending fibers extend caudally through the medial nucleus, terminating within the dorsal portion of this nucleus (Figs. 7B-E, 8B), as well as continuing caudally to terminate within the caudal octavolateralis nucleus (Fig. $7 F)$. Ascending fibers of the PLLN extend rostrally through the dorsal portion of the medial component of the eminentia granularis pars lateralis (Fig. 7A). Some fibers continue rostrally, terminating in the ipsilateral and contralateral eminentia granularis pars rostralis and pars dorsalis, as well as the ispilateral and contralateral ventral granular layer of the cerebellum (Figs. 6, 7A, 8C), in a manner similar to the projections of the ventral root of the ALLN. However, first-order fibers and terminals of the PLLN remain confined to the most dorsal portions of the those cell groups, whereas those of the ALLN are confined to the medial portions of the same cell groups, directly ventral to the projections of the PLLN. Neither ALLN or PLLN projections were observed to the most ventral portions of the eminentiae granularis or ventral granular layers of the cerebellar corpus.

Large neurons, located bilaterally in the same rostrocaudal plane as the branchiomeric motor nuclei, label retrogradely (Figs. 5D, 7D) following application of HRP to either the ALLN or PLLN. Cells labeled by HRP in the ALLN are located rostral to those labeled by HRP in the PLLN. All of these cells appear to be afferent to the lateralis nerves, and separate populations appear to project into each lateralis nerve.

\section{DISCUSSION}

The central projections of the lateral line nerves in $\mathbf{S c a}$ phirhynchus are similar to those reported in chondrichthians and other fishes, but there are a number of significant differences. The ALLN in sturgeons, like the ALLN in chrondrichthians, is divided into dorsal and ventral roots. The fibers of the dorsal root enter the medulla and terminate in the ipsilateral dorsal octavolateralis nucleus and in a more rostrally located granule cell population (dorsal granular component of the pars lateralis of the eminentia granularis). The trajectory and termination of the dorsal root fibers are similar to those reported by Koester ('83) in the clearnose skate (Raja eglanteria).

The fibers of the ventral root of the anterior lateral line nerve, as well as the fibers of the posterior lateral line nerve, enter the medulla and terminate primarily in the 


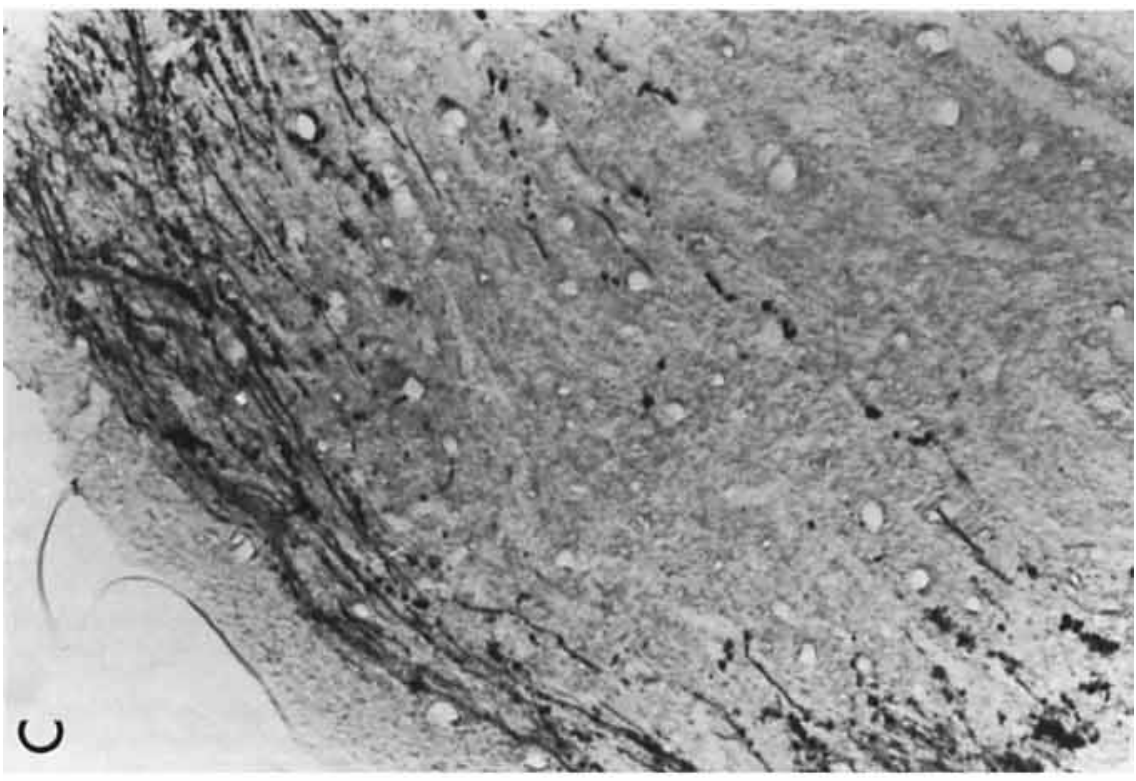

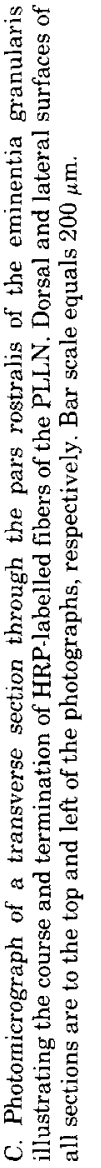

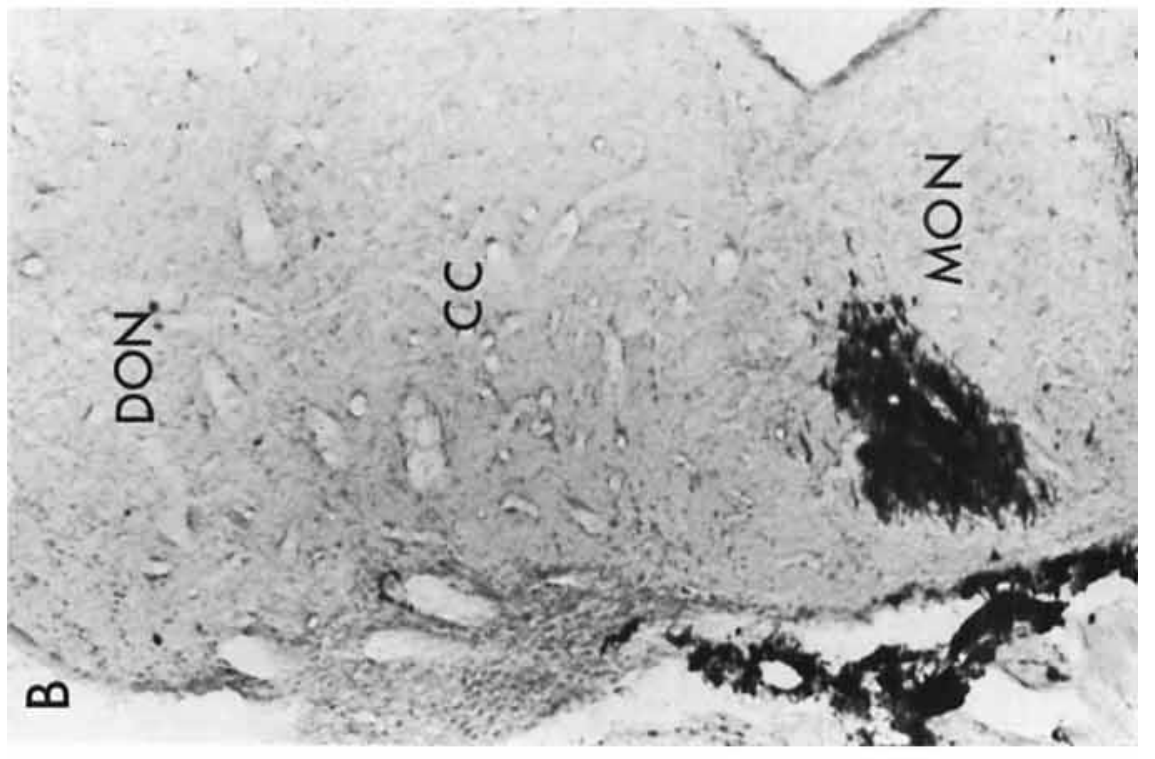

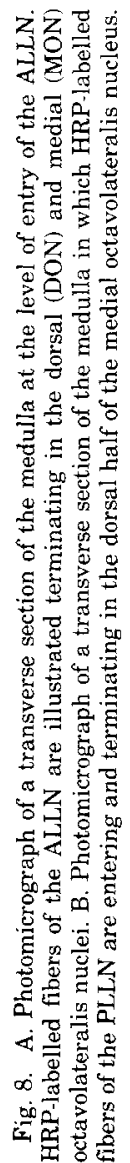

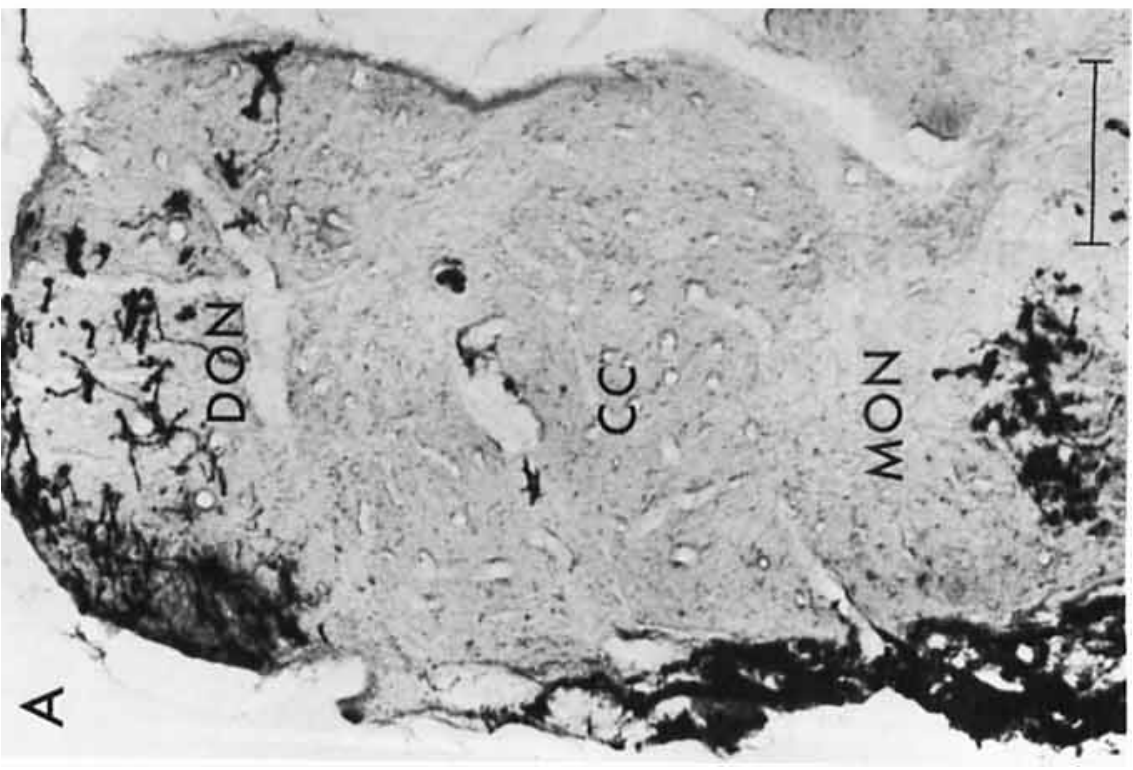


ipsilateral medial octavolateralis nucleus, more rostrally in a granule cell population (medial granular component of the pars lateralis of the eminentia granularis), and in a more caudal population (caudal octavolateralis nucleus). Again, similar projections have been reported in Raja (Koester, '83). Given these similarities in the primary projections of the lateral line nerves in sturgeons and Raja it is likely that the dorsal octavolateralis nucleus in sturgeons is an electroreceptive nucleus and that electroreceptive and mechanoreceptive inputs are spatially separated within the medulla of sturgeons as they are in skates.

The topography of the lateral line projections into the medial octavolateralis nucleus of Raja (Koester, '83) does differ from that in Scaphirhynchus. In Raja the anterior and posterior lateral line nerves terminate laterally and medially, respectively, whereas in Scaphirhynchus the anterior and posterior lateral line nerves terminate ventrally and dorsally, respectively. At present, there is no obvious explanation for these differences.

The projections of the primary lateral line fibers into the eminentia granularis in sturgeons and their topographic organization within the eminentia and octavolateralis nuclei are similar to that described in the bony fish Amia (McCormick, ' 81 ). In both these fish, the fibers of the anterior lateral line nerve terminate ventral to those of the posterior lateral line nerve within the eminentia granularis and the medial and caudal octavolateralis nuclei. Preliminary experiments in Scaphirhynchus (New and Northcutt, unpublished observations) indicate that the most ventral portion of the eminentia granularis is occupied by ascending fibers of the octaval nerve as was described in Amia.

Finally, a unique feature of the primary projections of the lateral line nerves in Scaphirhynchus is a bilateral projection to the ventral granular layers of the cerebellar corpus. This projection extends caudally through the corpus of the cerebellum, and rostrally through the valvula, and maintains the same topographic organization observed within the eminentia granularis. Also unusual is the projection to the contralateral eminentia granularis, which also maintains the same topographic organization. A bilateral projection to the mechanoreceptive lateral line lobe of mormyrid teleosts has been reported (Maler et al., '73; Bell and Russell, '78). Given the taxonomic position of these fishes and the known distribution of a bilateral projection of primary mechanoreceptive fibers in bony fishes, it is likely that crossed primary lateral line nerve projections have evolved independently in sturgeons and mormyrid teleosts.

Given the presence in sturgeons of ampullary organs on the head, their innervation of fibers of the anterior lateral line nerve, and separate medullary targets for fibers of the dorsal and ventral roots of the ALLN, as well as the presence of the same characters in chondrichthians, it is likely that these neural characters were present in the ancestral jawed fishes that gave rise to both chrondrichthians and chondrostean bony fishes. If so, further studies of the higherorder centers and pathways of the lateral line system in sturgeons would yield results similar to those already reported for chondrichthians (Boord and Northcutt, '82).

\section{ACKNOWLEDGMENTS}

We are grateful for the reliable and friendly service of $\mathrm{Mr}$. Eldon Saeugling, who collected the sturgeons, and $\mathrm{Mr}$. Harry Sloan, who was responsible for some of the histological preparations. Mary Sue Northcutt assisted in several phases of the research and preparation of the manuscript. This work was supported in part by USPHS grants NS11006 and EY02485.

\section{LITERATURE CITED}

Bell, C.C., and C.J. Russell (1978) Termination of electroreceptor and me chanical lateral line afferents in the mormyrid acousticolateral area. I. Comp. Neurol. 182:367-382.

Bodznick, D., and R.G. Northcutt (1980) Segregation of electro- and mechanoreceptive inputs to the elasmobranch medulla. Brain Res. 195:313-321.

Boord, R.L., and C.B.G. Campbell (1977) Structural and functional organization of the lateral line system of sharks. Am. Zool. 17:431-441.

Boord, R.L., and R.G. Northcutt (1982) Ascending lateral line pathways to the midbrain of the clearnose skate, Raja eglanteria. J. Comp. Neurol. 207:274-282.

Coleman, D.R., F. Scalia, and E. Cabrales (1976) Light and electron microscopic observations on the anterograde transport of horseradish peroxidase in the optic pathway in the mouse and rat. Brain Res., 102:156163.

Hocke Hoogenboom, K.J. (1929) Das Gehirn von Polyodon folium Lacep. Z. Mikr.-Anat. Forsch. 18:311-393.

Johnston, J.B. (1901) The brain of Acipenser. Zool. Jb. Anat. Abt. 15:59-260.

Jorgensen, J.M. (1980) The morphology of the Lorenzinian ampullae of the sturgeon Acipenser ruthenus. Acta Zool. (Stockholm) 61:87-92.

Jorgensen, J.M., A. Flock, and J. Wersall (1972) The Lorenzian ampullae of Polydon spathulae. Z. Zellforsch. 130:362-377.

Koester, D.M. (1983) The octavolateralis system of the clearnose skate, Raja eglanteria. I. Central projections of the lateral line nerves. J. Comp. Neurol. (in press).

Koester, D.M., and R.L. Boord (1978) The central projections of first order anterior lateral line neurons of the clearnose skate Raja eglanteria. Am. Zool. 18:587.

Larsell, O. (1967) The Comparative Anatomy and Histology of the Cerebellum from Myxinoids through Birds. Minneapolis: The University of Minnesota Press.

Maler, L., H.J. Karten, and J.V.L. Bennett (1973) The central connections of the posterior lateral line nerve of Gnathonemus petersii. J. Comp. Neurol. 151:57-66.

Marinelli, W., and A. Strenger (1973) Vergleichende Anatomie und Morphologie der Wirbeltiere. IV. Lieferung. Vienna: Franz Deuticke.

McCormick, C.A. (1981) Central projections of the lateral line and eighth nerves in the bowfin, Amia calva. J. Comp. Neurol. 197:1-15.

McCormick, C.A. (1982) The organization of the octavolateralis area in actinopterygian fishes: A new interpretation. J. Morphol. 171:159-181.

Mesulam, M.-M. (1978) Tetramethyl benzidine for horseradish peroxidase neurohistochemistry: A non-carcinogenic blue reaction product with superior sensitivity for visualizing neural afferents and efferents. J. Histochem. Cytochem. 26:106-117.

Murray, R.W. (1960) The response of the ampullae of Lorenzini of elasmobranchs to mechanical stimulation. J. Exp. Biol, 37:417-424.

Pearson, A.A. (1936) The acoustico-lateral centers and the cerebellum, with fiber connections, of fishes. J. Comp. Neurol. 65:201-294.

Teeter, J.H., R.B. Szamier, and M.V.L. Bennett (1980) Ampullary electroreceptors in the sturgeon Scaphirhynchus platorynchus. J. Comp. Physiol. $138: 213-223$

Weizel, G.F. (1978) The integument and caudal filament of the shovelnose sturgeon, Scaphirhynchus platorynchus. Am. Midl. Nat. 100:179-189.

Wiitanen, J.R. (1969) Selective impregnation of degenerating axons and axon terminals in the CNS of the monkey (Macaca mulatta). Brain Res. 14:546-548. 